\title{
Energy stable and high-order-accurate finite difference methods on staggered grids
}

Ossian O'Reilly, Tomas Lundquist, Eric M. Dunham and J an Nordström

The self-archived version of this journal article is available at Linköping University Electronic Press:

http:/ / urn.kb.se/ resolve?urn=urn:nbn:se:liu:diva-139343

N.B.: When citing this work, cite the original publication.

O'Reilly, O., Lundquist, T., Dunham, E. M., Nordström, J ., (2017), Energy stable and high-orderaccurate finite difference methods on staggered grids, J ournal of Computational Physics, 346, 572589. https:// dx.doi.org/ 10.1016/j.jcp.2017.06.030

Original publication available at:

https:// dx.doi.org/ 10.1016/j.jcp.2017.06.030

Copyright: Elsevier

http:// www.elsevier.com/

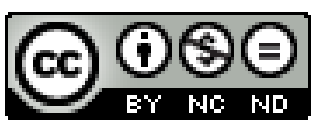




\title{
Energy stable and high-order-accurate finite difference methods on staggered grids
}

\author{
Ossian O’Reilly ${ }^{\mathrm{a}, \mathrm{b}, *}$, Tomas Lundquist ${ }^{\mathrm{b}}$, Eric M. Dunham ${ }^{\mathrm{a}, \mathrm{c}}$, Jan \\ Nordström ${ }^{\mathrm{b}}$ \\ ${ }^{a}$ Department of Geophysics, Stanford University, CA 94305-2215 \\ ${ }^{b}$ Division of Computational Mathematics, Department of Mathematics, Linköping \\ University, SE-581 83 Linköping, Sweden \\ ${ }^{c}$ Institute for Computational and Mathematical Engineering, Stanford University, \\ Stanford, CA, USA
}

\begin{abstract}
For wave propagation over distances of many wavelengths, high-order finite difference methods on staggered grids are widely used due to their excellent dispersion properties. However, the enforcement of boundary conditions in a stable manner and treatment of interface problems with discontinuous coefficients usually pose many challenges. In this work, we construct a provably stable and high-order-accurate finite difference method on staggered grids that can be applied to a broad class of boundary and interface problems. The staggered grid difference operators are in summation-by-parts form and when combined with a weak enforcement of the boundary conditions, lead to an energy stable method on multiblock grids. The general applicability of the method is demonstrated by simulating an explosive acoustic source, generating waves reflecting against a free surface and material discontinuity.
\end{abstract}

\section{Introduction}

Some of the most popular numerical methods for wave propagation are high-order finite difference (FD) methods on staggered grids. In computational electromagnetism, these methods are based on the traditional secondorder Yee-scheme [1] and extended to high-order via either explicit or implicit

\footnotetext{
${ }^{*}$ Corresponding author

Email address: ooreilly@stanford.edu (Ossian O'Reilly)
} 
(compact) difference operators [2, 3, 4, 5, 6. Similar methods have been developed and applied to other areas of wave propagation such as computational seismology and acoustics. In computational seismology, the velocitystress formulation for solving the elastic wave equation is commonly used [7, 8, 9, 10]. Although a few decades have past since the introduction of staggered grid methods, they are still actively and widely used today. This widespread success is because of their simplicity, accuracy, computational efficiency, and ability to scale for large problems.

Compared to low-order methods, high-order methods have small dispersion errors which enable accurate wave propagation over long distances using relatively few grid points per wavelength [11]. The arrangement of variables in the grid can also have a strong impact on the accuracy. Unlike in a collocated grid where all variables are stored at the same grid points, in a staggered grid a variable is shifted by half of a grid spacing with respect to each other. The first derivative is computed more accurately on a staggered grid and staggered grid methods have generally been found to be more accurate than collocated grid methods [12, 13, 14]. Staggering in time can also be used to further improve accuracy and reduce storage costs [15].

Despite the success of these methods, they have three fundamental drawbacks, two of which have attracted considerable attention. First, curved geometries have to be approximated by staircased grids. Second, the boundary conditions needed at a material interface, where the solution can be discontinuous or non-smooth, are enforced by differentiating across the interface. Both of these issues degrade the accuracy of the numerical solution. Degradation to first-order-accurate and even nonconvergent approximations have been reported and analyzed by many authors [16, 17, 18, 19]. Significantly less attention has been paid to the construction of provably stable methods. The lack of a provably stable method is a major drawback that must not be overlooked. The most dreadful instabilities are elusive in nature. While a numerical solution is typically compared against analytic solutions and other codes by performing grid convergence studies, there is no guarantee that the numerical solution is stable for other types of problems. It is also nontrivial to numerically investigate stability by computing eigenvalues of the large-scale problems often encountered in practice. Therefore, only a proof of stability suffices for producing reliable numerical solutions.

A vast number of solutions that address the aforementioned accuracy issues while maintaining the original simplicity have been proposed. In [20], a planar interface treatment that achieves fourth-order accuracy using one- 
sided differences was developed. The need for staircased grid approximations can be overcome by using curvilinear mappings. Methods have been developed for both orthogonal curvilinear grids [21] as well as general curvilinear grids [22, 6, 23, 24]. A more direct way to maintain simplicity is to use immersed interface or embedded methods. These methods enforce the jump conditions at the interface by embedding it in the Cartesian grid and only modifying the weights in the FD stencils near the interface. Immersed methods have been considered by an extensive number of authors, see e.g., [25, 26, 27, 28, 29, 30], and the references therein. However, immersed methods are low-order-accurate. A third option is to combine unstructured and structured grids to form a hybrid method. The hybrid method is most useful when the curved geometry only occupies a small region of the computational domain. Finite element methods (FEM) have been coupled to FD [31, 32]. FEM is used on an unstructured grid in a region with complex geometry and is coupled to the FD method used on a surrounding Cartesian grid. Similar ideas have also been applied using overlapping or composite grid methods [26].

In all of the previously mentioned cases, only strongly enforced boundary conditions have been considered and stability has not been analyzed beyond von Neumann stability (with the exception of [17]). The purpose of the present study is to construct a provably stable high-order method on a staggered grid using weakly enforced boundary conditions. There are many advantages with weakly enforced boundary conditions. The most important advantage is the straightforward path to stability for high-order methods in summation-by-parts form (see discussion below).

In practical calculations, weakly enforced boundary conditions often simplify the implementation. One example is in tsunami generation due to earthquakes, which can be modeled by prescribing traction-free boundary conditions on a linearized moving sea surface [33]. Another example is the simulation of earthquake rupture dynamics, which requires the coupling of elasticity to nonlinear friction laws at an interface where some of the fields can be discontinuous [34, 35, 36, 37]. In computational electromagnetics, frequency dependent dispersive interfaces can be treated by using time dependent jump conditions [38. Weakly enforced boundary conditions are also a fundamental component of the Discontinuous Galerkin (DG) methods. In DG methods, penalty terms known as numerical fluxes are used to enforce boundary conditions and achieve element-to-element coupling (and also stability). 
Similar ideas are presented in summation-by-parts (SBP) methods that use a weak enforcement of boundary conditions via the simultaneous approximation term (SAT) penalty technique. SBP methods were originally developed for FD [39, 40, 41, 42], but several other methods have been shown to be in this form, including finite volume, spectral methods [43, discontinuous Galerkin spectral element methods [4], and flux reconstruction methods [45]. SBP operators satisfy a summation-by-parts property that mimics integration by parts. This property is essential for obtaining an energy estimate, but it is in its standard form restricted to collocated grids. In this work, we extend the SBP-SAT approach to staggered grids by identifying a new SBP property. Given this new SBP property, we construct an energy stable scheme for the acoustic wave equation with multiblock coupling. Due to the general nature of the approach, it can also be applied to other types of wave equations such as the elastic wave equation, or Maxwell's equations.

There are also many advantages of extending the SBP-SAT approach to staggered grids. With this development in hand, one can construct stable, dual consistent difference approximations which are important for optimization problems [46, 47]. While the method in the present study is developed on Cartesian grids, one can gain geometric flexibility by coupling to other SBP methods, e.g., finite volume methods [48, 49, 36], or DG methods [50]. The method developed in the present study may also be applied to other domains that use staggered grids. For example, in the incompressible Navier-Stokes equations a staggered grid is often used to overcome spurious oscillations in the pressure field [51]. To take advantage of many of these benefits, one only has to modify the boundary procedures in existing staggered grid FD codes.

Generally speaking, the part of an FD code that performs the bulk of the computational work consists of compute kernels responsible for the application of the FD stencils in the interior of the domain. For this reason, the compute kernels are usually heavily optimized and fine-tuned for the specific architecture in mind. The software optimization required to achieve the desired level of throughput can consume both significant effort and time. Since the SBP-SAT method in the present study uses the same interior FD stencils as found in numerous codes, all interior compute kernels can be reused without modification. However, the boundary procedures need to be replaced. On the other hand, since the boundary computations constitute such a small fraction of the computational cost, these procedures do not require the same level of aggressive optimization.

This paper is organized as follows. Section 2 defines SBP operators on 
staggered grids. Sections 34 use these definitions to solve the wave equation in one and two dimensions subject to linear boundary conditions. Section 5 is dedicated to performing a series of numerical experiments to assess the accuracy of the new SBP staggered grid operators and showcase some of their capabilities. In particular, we consider singular source terms to set off explosions, and solve problems with material discontinuities. Finally, in Section 6] conclusions are drawn.

\section{Definitions}

In this section, we construct SBP high-order finite difference operators on staggered grids. We discretize the interval $x \in[0,1]$ using the grid points

$$
x_{j}=j h, 0 \leq j \leq N, \quad \text { and } \quad x_{j-1 / 2}=(j-1 / 2) h, 1 \leq j \leq N,
$$

where $h=1 / N$ is the grid spacing. Let the staggered grids be defined as

$$
\begin{aligned}
& x_{+}=\left[x_{0}, x_{1}, \ldots, x_{N}\right]^{T} \in \mathbb{R}^{N+1} \\
& x_{-}=\left[x_{0}, x_{1 / 2}, \ldots, x_{N-1 / 2}, x_{N}\right]^{T} \in \mathbb{R}^{N+2} .
\end{aligned}
$$

Note that, both grids contain the endpoints $x_{0}=0$ and $x_{N}=1$.

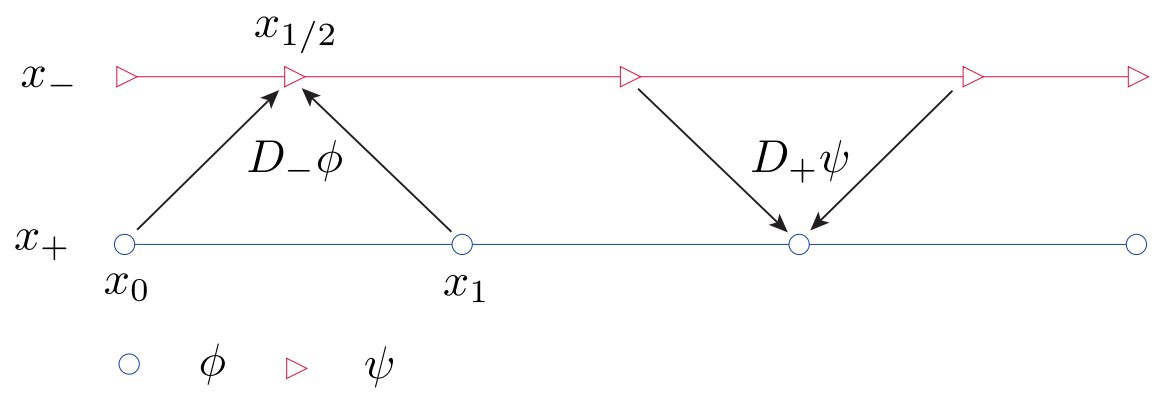

Figure 1: Illustration of second-order accurate difference approximations on staggered grids.

SBP staggered grid finite difference operators are constructed by using central difference approximations at interior points and one-sided difference approximations at boundary points. For example, second-order accuracy in the interior is given by the standard central difference approximations

$$
\left.\frac{d \psi}{d x}\right|_{x_{i}} \approx \frac{\psi_{i+1 / 2}-\psi_{i-1 / 2}}{h},\left.\frac{d \phi}{d x}\right|_{x_{i-1 / 2}} \approx \frac{\phi_{i}-\phi_{i-1}}{h}
$$


for smooth grid functions $\psi$ and $\phi$ defined on $x_{-}$and $x_{+}$, respectively. Of course, higher-order differences can be used instead.

We store the difference approximations in matrices $D_{+} \in \mathbb{R}^{(N+1) \times(N+2)}$, $D_{-} \in \mathbb{R}^{(N+2) \times(N+1)}$. The difference operator $D_{+}$acts on a grid function defined on $x_{-}$, but approximates its derivative on $x_{+}$. Similarly, the difference operator $D_{-}$acts on a grid function defined on $x_{+}$, but approximates its derivative on $x_{-}$. Figure 1 demonstrates how the difference operators act on the grid functions for second-order accuracy. SBP staggered grid operators are defined as follows.

Definition 1. The difference operators $D_{+}=P_{+}^{-1} Q_{+}$and $D_{-}=P_{-}^{-1} Q_{-}$are $S B P$ staggered grid difference operators if the following properties hold:

(i) (Accuracy) The order of accuracy is s for boundary points and $2 s$ for interior points. Given monomials $x_{+}^{k}$ and $x_{-}^{k}$, we have the accuracy relations

$$
D_{+} x_{-}^{k}=k x_{+}^{k-1}, D_{-} x_{+}^{k}=k x_{-}^{k-1}, k=0,1, \ldots, s .
$$

(ii) (Norm) $P_{+}$and $P_{-}$are diagonal and positive definite matrices defining the respective discrete $L_{2}$ norms

$$
\begin{aligned}
& \|\phi\|_{h}^{2}=\phi^{T} P_{+} \phi=h \sum_{j=0}^{N} \alpha_{j} \phi_{j}^{2} \approx \int_{0}^{1} \phi^{2} d x \\
& \|\psi\|_{h}^{2}=\psi^{T} P_{-} \psi=h \beta_{0} \psi_{0}^{2}+h \beta_{N} \psi_{N}^{2}+h \sum_{j=1}^{N} \beta_{j} \psi_{j-1 / 2}^{2} \approx \int_{0}^{1} \psi^{2} d x,
\end{aligned}
$$

for smooth grid functions $\phi$ defined on $x_{+}$and $\psi$ defined on $x_{-}$.

(iii) (SBP-property) The matrices $Q_{+}$and $Q_{-}$satisfy the $S B P$ property

$$
Q_{+}+Q_{-}^{T}=B_{+}=B_{-}^{T}=B
$$

where the matrix $B$ restricts the grid functions to the boundary: $\phi^{T} B \psi=$ $\phi_{N} \psi_{N}-\phi_{0} \psi_{0}$.

The operators are built in such way that the properties (i)-(iii) in Definition 1 are satisfied by construction. 
When we refer to the order of accuracy of the SBP operators, we refer to the interior order of accuracy. For example, a pair of second-order accurate SBP staggered grid operators is

$$
\begin{aligned}
& Q_{+}=\left[\begin{array}{rrrrrrr}
-\frac{1}{2} & \frac{1}{4} & \frac{1}{4} & & & & \\
-\frac{1}{2} & -\frac{1}{4} & \frac{3}{4} & & & & \\
& & -1 & 1 & & & \\
& & & \ddots & \ddots & & \\
& & & & -1 & 1 & \\
& & & & -\frac{3}{4} & \frac{1}{4} & \frac{1}{2} \\
& & & & -\frac{1}{4} & -\frac{1}{4} & \frac{1}{2}
\end{array}\right] \\
& Q_{-}=\left[\begin{array}{rrrrrrrr}
-\frac{1}{2} & \frac{1}{2} & & & & & & \\
-\frac{1}{4} & \frac{1}{4} & & & & & & \\
-\frac{1}{4} & -\frac{3}{4} & 1 & & & & & \\
& & -1 & 1 & & & & \\
& & & \ddots & \ddots & & & \\
& & & & -1 & 1 & & \\
& & & & & -1 & \frac{3}{4} & \frac{1}{4} \\
& & & & & & -\frac{1}{4} & \frac{1}{4} \\
& & & & & & -\frac{1}{2} & \frac{1}{2}
\end{array}\right],
\end{aligned}
$$

and

$$
\begin{aligned}
& P_{+}=\operatorname{diag}\left(\left[\frac{1}{2}, 1,1, \ldots, 1, \frac{1}{2}\right]\right) h \\
& P_{-}=\operatorname{diag}\left(\left[\frac{1}{2}, \frac{1}{4}, \frac{5}{4}, 1, \ldots, 1, \frac{5}{4}, \frac{1}{4}, \frac{1}{2}\right]\right) h .
\end{aligned}
$$

This approximation uses the second order central derivatives (1). Near and on each of two boundaries, two one-sided stencils are used for constructing $D_{+}$and three one-sided stencils for constructing $D_{-}$.

To derive the SBP operators above, we introduced a $2 \times 3$ block of unknown coefficients $q_{i j}$ in the top left corner of $Q_{+}, 2$ unknown coefficients $\alpha_{i}$, $i=0,1$, in $P_{+}$, and 3 unknown coefficients $\beta_{j}, j=0,1,2$, in $P_{-}$. All of these unknown coefficients are determined by satisfying the accuracy conditions (2), more details are given below. In the interior, $Q_{+}$has a banded structure given by the stencil $(-1,1)$, and the coefficients in $P_{+}$and $P_{-}$are equal to unity. 
The structure of $Q_{+}$for high-order approximations follow along the same lines. For example, a fourth-order operator with a block $4 \times 5$ can be constructed by assuming the structure:

$$
Q_{+}=\left[\begin{array}{cccccccccc}
q_{00} & q_{01} & q_{02} & q_{03} & q_{04} & 0 & 0 & 0 & 0 & 0 \\
q_{10} & q_{11} & q_{12} & q_{13} & q_{14} & 0 & 0 & 0 & 0 & 0 \\
q_{20} & q_{21} & q_{22} & q_{23} & q_{24} & 0 & 0 & 0 & 0 & 0 \\
q_{30} & q_{31} & q_{32} & q_{33} & q_{34} & 1 / 24 & 0 & 0 & 0 & 0 \\
0 & 0 & 0 & 1 / 24 & -9 / 8 & 9 / 8 & -1 / 24 & 0 & 0 & 0 \\
0 & 0 & 0 & 0 & -1 / 24 & -q_{34} & -q_{33} & -q_{32} & -q_{31} & -q_{30} \\
0 & 0 & 0 & 0 & 0 & -q_{24} & -q_{23} & -q_{22} & -q_{21} & -q_{20} \\
0 & 0 & 0 & 0 & 0 & -q_{14} & -q_{13} & -q_{12} & -q_{11} & -q_{10} \\
0 & 0 & 0 & 0 & 0 & -q_{04} & -q_{03} & -q_{02} & -q_{01} & -q_{00}
\end{array}\right] .
$$

To conserve space, only one interior stencil is shown. The matrix $Q_{-}$is directly obtained from $Q_{-}=-Q_{+}^{T}+B^{T}$ in (4).

In general, $Q_{+}$has a block of $m \times n$ unknowns. By multiplying the two equations in (2) with $P_{+}$and $P_{-}$, respectively, we obtain a linear equation system for the unknowns $q_{i j}, \alpha_{i}, \beta_{j}, 0 \leq i \leq(m-1), 0 \leq j \leq(n-1)$. The solution to this system (if it exists) is usually non-unique. Any free parameters that remain can be optimized for accuracy or spectral radius. When solving (2) it is important to ensure that quadrature weights in $P_{+}$and $P_{-}$ are positive, since otherwise condition (i) in Definition 1 is violated. We solve (2) symbolically using SymPy, a Python library for symbolic mathematics 52 .

In the present study we have derived staggered SBP operators for orders 2, 4, and 6. These operators are available at github.com/ooreilly/sbp. The free parameters have been chosen by optimizing for accuracy as proposed in 53 .

\section{Analysis in one dimension}

In this section, we use the SBP staggered grid operators to construct a stable, semi-discrete approximation of the wave equation. Stability is shown by deriving a discrete energy estimate and weakly enforcing the boundary conditions. 


\subsection{Continuous problem}

As a model problem, consider the wave equation in one dimension:

$$
\frac{\partial p}{\partial t}+\frac{\partial u}{\partial x}=0, \frac{\partial u}{\partial t}+\frac{\partial p}{\partial x}=0, \quad 0 \leq x \leq 1, t \geq 0
$$

We apply the energy method [54], which leads to

$$
\frac{1}{2} \frac{d}{d t}\left(\|p\|^{2}+\|u\|^{2}\right)=-\left.p u\right|_{0} ^{1}
$$

where $\|p\|^{2}=\int_{0}^{1} p^{2} d x$. For simplicity, consider only the boundary at $x=0$. The number and form of boundary conditions are determined by a diagonalization. The right-hand side in (8) is written as a quadratic form:

$$
\left.p u\right|_{0}=\left.\frac{1}{2} U^{T} A U\right|_{0}, U=\left[\begin{array}{l}
p \\
u
\end{array}\right], A=\left[\begin{array}{ll}
0 & 1 \\
1 & 0
\end{array}\right],
$$

which is diagonalized by

$$
A=X \Lambda X^{T}, X=\frac{1}{\sqrt{2}}\left[\begin{array}{cc}
-1 & 1 \\
1 & 1
\end{array}\right], \Lambda=\left[\begin{array}{cc}
-1 & 0 \\
0 & 1
\end{array}\right] .
$$

By inserting (10) into (8), we obtain

$$
\frac{d}{d t}\left(\|p\|^{2}+\|u\|^{2}\right)=\left.w^{T} \Lambda w\right|_{0}=\left.\left(w^{+}\right)^{2}\right|_{0}-\left.\left(w^{-}\right)^{2}\right|_{0},
$$

where $w=\left[w^{-}, w^{+}\right]^{T}, w^{+}=\left(X^{+}\right)^{T} U$ and $w^{-}=\left(X^{-}\right)^{T} U$ are the incoming and outgoing characteristic variables, propagating into and out of the domain, respectively. Furthermore, $\Lambda=\operatorname{diag}\left(\left[\Lambda^{-}, \Lambda^{+}\right]^{T}\right)$ contains the positive and negative eigenvalues, and $X=\left[X^{-}, X^{+}\right]$are the eigenvectors. The characteristic variables are

$$
w^{-}=\frac{u-p}{\sqrt{2}} \quad \text { and } \quad w^{+}=\frac{u+p}{\sqrt{2}} .
$$

The number of boundary conditions needed is equal to the number of positive eigenvalues. Since $\Lambda^{+}$contains precisely one positive eigenvalue, one boundary condition must be imposed. We consider a linear homogeneous boundary condition of the form

$$
\left.L^{T} U\right|_{0}=\left.w^{+}\right|_{0}-\left.r w^{-}\right|_{0}=0,
$$


for a given reflection coefficient $r$, where the boundary operator is

$$
L=\frac{1}{\sqrt{2}}[1+r, 1-r]^{T}
$$

Finally, by imposing the boundary condition, (11) becomes

$$
\begin{aligned}
\frac{d}{d t}\left(\|u\|^{2}+\|p\|^{2}\right)=\left.w^{T} \Lambda w\right|_{0} & =\left.\left(w^{+}\right)^{T} \Lambda^{+} w^{+}\right|_{0}+\left.\left(w^{-}\right)^{T} \Lambda^{-} w^{-}\right|_{0} \\
& =-\left.\left(1-r^{2}\right)\left(w^{-}\right)^{2}\right|_{0},
\end{aligned}
$$

and we have an estimate for $|r| \leq 1$.

\subsection{The semi-discrete approximation}

Next, we use the results from the continuous analysis to construct a stable, semi-discrete approximation of the problem (7). While we present the method for the constant coefficient case with the wave speed set to unity, a semi-discrete approximation and corresponding analysis for the variable coefficient case with a multiblock interface is presented in Appendix A.

Consider the semi-discrete approximation

$$
\begin{aligned}
& u_{t}+D_{+} p=\sigma_{1} P_{+}^{-1} e_{0+}\left(w_{0}^{+}-r w_{0}^{-}\right) \\
& p_{t}+D_{-} u=\sigma_{2} P_{-}^{-1} e_{0-}\left(w_{0}^{+}-r w_{0}^{-}\right)
\end{aligned}
$$

In (16), the grid functions $u$ and $p$ are stored on the $x_{+}$and $x_{-}$grids, respectively. The right-hand side contains penalty terms that weakly enforce the boundary condition (13). The penalty terms are restricted to the boundary using: $u_{0}=e_{0+}^{T} u$ and $p_{0}=e_{0-}^{T} p$. The parameters $\sigma_{1}$ and $\sigma_{2}$ will be determined for stability. For simplicity, as in the previous section, we have neglected the boundary condition at $x=1$. The characteristic variables $w^{+}$ and $w^{-}$are defined using (12). This definition is possible because both $p$ and $v$ are defined at the boundary.

Proposition 1. The semi-discrete approximation (16) of (7) with boundary condition (13) is stable if

$$
\sigma_{1}=-\frac{1}{\sqrt{2}} \quad \text { and } \quad \sigma_{2}=-\frac{1}{\sqrt{2}} .
$$


Proof. We apply the discrete energy method to (16). By using the SBP property (4), we get

$$
\frac{1}{2} \frac{d}{d t}\left(\|p\|_{h}^{2}+\|u\|_{h}^{2}\right)=\frac{1}{2} U_{0}^{T} A U_{0}+U_{0}^{T} \Sigma\left(w_{0}^{+}-r w_{0}^{-}\right),
$$

where $\|p\|_{h}^{2}=p^{T} P_{-} p$ and $\|u\|_{h}^{2}=u^{T} P_{+} u$, and $p_{0} v_{0}$ has been replaced by $U_{0}^{T} A U_{0} / 2$ using (9), and $\Sigma=\left[\sigma_{1}, \sigma_{2}\right]^{T}$. By diagonalizing $A$ using (10) and performing the substitution $W=X^{T} U$, we get

$$
\frac{1}{2} \frac{d}{d t}\left(\|p\|_{h}^{2}+\|u\|_{h}^{2}\right)=\frac{1}{2}\left(w_{0}^{+}\right)^{2}-\frac{1}{2}\left(w_{0}^{-}\right)^{2}+W_{0}^{T} X^{T} \Sigma\left(w_{0}^{+}-r w_{0}^{-}\right) .
$$

The choice $\Sigma=-X \Lambda^{+}=-[1,1]^{T} / \sqrt{2}$ yields $W_{0}^{T} X^{T} \Sigma=-w_{0}^{+}$, and

$$
\begin{aligned}
\frac{d}{d t}\left(\|p\|_{h}^{2}+\|u\|_{h}^{2}\right) & =\left(w_{0}^{+}\right)^{2}-\left(w_{0}^{-}\right)^{2}-2 w_{0}^{+}\left(w_{0}^{+}-r w_{0}^{-}\right) \\
& =-\left(w_{0}^{+}\right)^{2}-\left(w_{0}^{-}\right)^{2}+2 r w_{0}^{+} w_{0}^{-}+r^{2}\left(w_{0}^{-}\right)^{2}-r^{2}\left(w_{0}^{-}\right)^{2} \\
& =-\left(1-r^{2}\right)\left(w_{0}^{-}\right)^{2}-\left(w_{0}^{+}-r w_{0}^{-}\right)^{2} \leq 0, \quad|r| \leq 1 .
\end{aligned}
$$

The result is similar to (15), but also includes numerical dissipation that vanishes with grid refinement.

\subsection{Energy conservative formulation}

In some cases it is desirable to have a strictly energy conservative semidiscrete approximation (assuming the boundary conditions are energy conservative). As an example, suppose we wish to enforce the boundary condition $p=0$ on $x=0$. Enforcing this boundary condition is accomplished by replacing the characteristic formulation (16) with

$$
\begin{aligned}
& \frac{d u}{d t}+D_{+} p=\sigma_{1} P_{+}^{-1} e_{0+} p, \\
& \frac{d p}{d t}+D_{-} u=\sigma_{2} P_{-}^{-1} e_{0-} p .
\end{aligned}
$$

By seeking $\sigma_{1}$ and $\sigma_{2}$ such that the semi-discrete approximation is stable we arrive at

$$
\frac{1}{2} \frac{d}{d t}\left(\|p\|_{h}^{2}+\|u\|_{h}^{2}\right)=\left(1+\sigma_{1}\right) p_{0} u_{0}+\sigma_{2} p_{0}^{2},
$$

which is non-positive if $\sigma_{1}=-1$ and $\sigma_{2} \leq 0$. For energy conservation we simply set $\sigma_{2}=0$. A similar procedure can be developed to enforce $v=0$ boundary condition with no energy dissipation. 


\section{Analysis in two dimensions}

In this section we extend the previous analysis to two dimensions.

\subsection{Continuous problem}

In two dimensions, the wave equation is

$$
\begin{array}{r}
\frac{\partial U}{\partial t}+A_{x} \frac{\partial U}{\partial x}+A_{y} \frac{\partial U}{\partial y}=0,(x, y) \in \Omega, t \geq 0, \\
U=\left[\begin{array}{l}
p \\
u \\
v
\end{array}\right], A_{x}=\left[\begin{array}{lll}
0 & 1 & 0 \\
1 & 0 & 0 \\
0 & 0 & 0
\end{array}\right], A_{y}=\left[\begin{array}{lll}
0 & 0 & 1 \\
0 & 0 & 0 \\
1 & 0 & 0
\end{array}\right] .
\end{array}
$$

The energy method applied to (23) leads to

$$
\frac{1}{2} \frac{d}{d t}\|U\|^{2}=-\oint_{\partial \Omega} p u_{n} d s
$$

where $u_{n}=u \hat{n}_{x}+v \hat{n}_{y}$ is the normal velocity, $\hat{n}=\left[\hat{n}_{x}, \hat{n}_{y}\right]^{T}$ is the outwardpointing unit normal associated with the boundary $\partial \Omega$, and $d s$ is the infinitesimal line element. To determine the number and form of boundary conditions, we first define $\bar{U}=\left[p,-u_{n}\right]^{T}$. Then $(24)$ becomes

$$
\frac{d}{d t}\|U\|^{2}=\oint_{\partial \Omega} \bar{U}^{T} A \bar{U} d s
$$

where $A$ is given in (9).

In two dimensions, the matrices $A_{x}$ and $A_{y}$ of the hyperbolic problem (23) are not simultaneously diagonalizable, and therefore characteristic variables are, strictly speaking, not defined. Instead we choose to diagonalize $A_{x} \hat{n}_{x}+$ $A_{y} \hat{n}_{y}$ and treat the problem as locally one-dimensional. We use the outward pointing normal on the boundary to locally define incoming and outgoing variables (into and out of the domain), which with a slight abuse of notation, we call characteristics. The incoming and outgoing characteristic variables are given by

$$
w^{+}=\frac{1}{\sqrt{2}}\left(p-u_{n}\right), w^{-}=-\frac{1}{\sqrt{2}}\left(p+u_{n}\right) .
$$

Again, we consider linear and homogeneous boundary conditions of the form

$$
\left.L \bar{U}\right|_{\partial \Omega}=\left.w^{+}\right|_{\partial \Omega}-\left.r w^{-}\right|_{\partial \Omega}=0
$$


where the boundary operator $L$ is given in (14). A convenient way to artificially truncate the domain is to enforce the non-reflective boundary condition given by $r=0$ in $(26)$. More accurate methods exist, but are beyond the scope of the present study. In applications, one may consider highorder non-reflective boundary conditions (see [55, 56] for a review), perfectly matched layers [57], or super-grid layers, combining artificial dissipation and grid stretching [58].

Once the boundary conditions have been enforced, (25) becomes

$$
\frac{d}{d t}\|U\|^{2}=-\oint_{\partial \Omega}\left(1-r^{2}\right)\left(w^{-}\right)^{2} d s
$$

which is bounded for $|r| \leq 1$.

\subsection{The semi-discrete approximation}

Consider the domain $\Omega=[0,1] \times[0,1]$ with boundaries denoted by the cardinal directions. The coordinate system is oriented such that the outward unit normal with respect to the north boundary is in the positive $y$-direction and so forth. A semi-discrete approximation of (23) enforcing the boundary condition 26 on the south boundary is (the other boundaries are treated in a analogous manner)

$$
\begin{aligned}
& \frac{d p}{d t}+\left(D_{x-} \otimes I_{y-}\right) u+\left(I_{x-} \otimes D_{y-}\right) v=\sigma_{1}\left(P_{x-} \otimes P_{y-}\right)^{-1} E_{S, p}^{T} H_{x-}\left(w_{S}^{+}-r w_{S}^{-}\right), \\
& \frac{d u}{d t}+\left(D_{x+} \otimes I_{y-}\right) p=0, \\
& \frac{d v}{d t}+\left(I_{x-} \otimes D_{y+}\right) p=\sigma_{2}\left(P_{x-} \otimes P_{y+}\right)^{-1} E_{S, v}^{T} H_{x-}\left(w_{S}^{+}-r w_{S}^{-}\right) .
\end{aligned}
$$

In (28), the grid functions $p, u$, and $v$ are stored in vectors having $y$ as the contiguous direction and their arrangement is shown in Figure 2, The difference operators are extended to two dimensions by using the Kronecker product $\otimes$. The identity matrices $I_{x+}$ etc. have the same dimension as the number of grid points in the coordinate direction, indicated by the subscript.

On the south boundary, the normal velocity is $u_{n}(x, 0, t)=-v(x, 0, t)$ and the characteristic variables are

$$
w_{S}^{+}=\frac{1}{\sqrt{2}}\left(p_{S}+v_{S}\right), w_{S}^{-}=-\frac{1}{\sqrt{2}}\left(p_{S}-v_{S}\right) .
$$



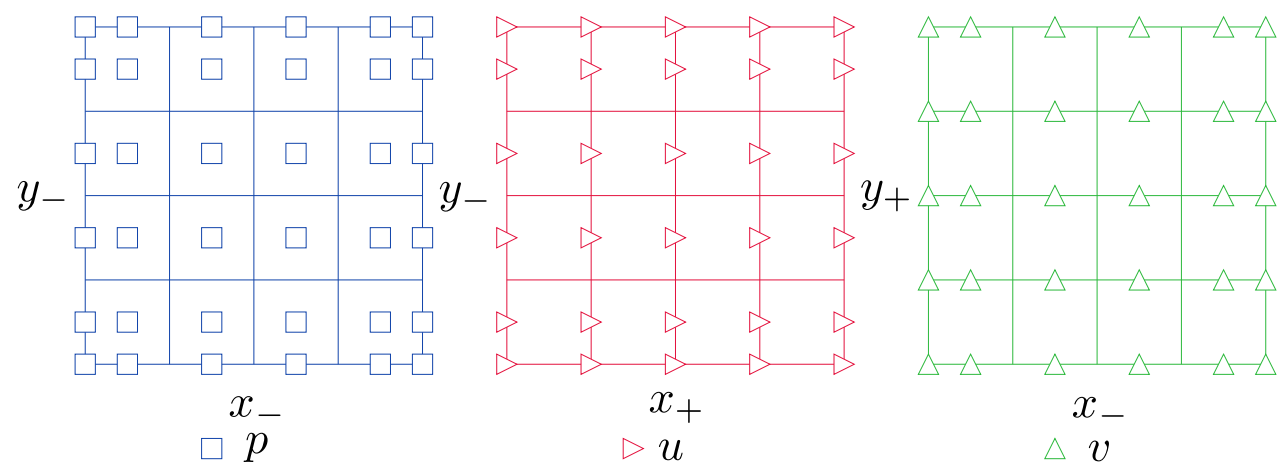

Figure 2: Staggered grid arrangement of unknowns for the semi-discrete approximation of the wave equation in 28 .

The restriction of the solution to the boundary is obtained using

$$
p_{S}=E_{S, p} p, v_{S}=E_{S, v} v, E_{S, p}=I_{x-} \otimes e_{y_{0}-}^{T}, E_{S, v}=I_{x-} \otimes e_{y_{0}+}^{T} .
$$

The stability of the semi-discrete approximation is addressed in the following proposition.

Proposition 2. The semi-discrete approximation (28) of (23) with boundary condition (26) is stable if

$$
\sigma_{1}=-\frac{1}{\sqrt{2}} \quad \text { and } \quad \sigma_{2}=-\frac{1}{\sqrt{2}} .
$$

Proof. As before, the result follows from applying the discrete energy method. By defining the discrete energy

$$
E_{h}=\frac{1}{2} p^{T}\left(P_{x-} \otimes P_{y-}\right) p+\frac{1}{2} u^{T}\left(P_{x+} \otimes P_{y-}\right) u+\frac{1}{2} v^{T}\left(P_{x-} \otimes P_{y+}\right) v
$$

and using the semi-discrete approximation (28), we have

$$
\begin{aligned}
\frac{d E_{h}}{d t}= & -p^{T}\left(Q_{x-} \otimes P_{y-}\right) u-p^{T}\left(P_{x-} \otimes Q_{y-}\right) v-u^{T}\left(Q_{x+} \otimes P_{y-}\right) p \\
& -v^{T}\left(P_{x-} \otimes Q_{y+}\right) p+\left(\sigma_{1} p_{S}+\sigma_{2} v_{S}\right)^{T} P_{x-}\left(w_{S}^{+}-r w_{S}^{-}\right) .
\end{aligned}
$$


By using the SBP property (4) and neglecting terms for all boundaries except the south boundary, we get

$$
\frac{d E_{h}}{d t}=p_{S}^{T} P_{x-} v_{S}+\left(\sigma_{1} p_{S}+\sigma_{2} v_{S}\right)^{T} P_{x-}\left(w_{S}^{+}-r w_{S}^{-}\right) .
$$

The choice $\sigma_{1}=\sigma_{2}=-1 / \sqrt{2}$ leads to

$$
\frac{d E_{h}}{d t}=-\frac{1}{2}\left(1-r^{2}\right)\left(w_{S}^{-}\right)^{T} P_{x-} w_{S}^{-}-\frac{1}{2}\left(w_{S}^{+}-r w_{S}^{-}\right)^{T} P_{x-}\left(w_{S}^{+}-r w_{S}^{-}\right) \leq 0,
$$

for $|r| \leq 1$

\section{Numerical experiments}

In this section, we conduct numerical experiments to assess the order of accuracy and dispersion properties using a manufactured solution. We also propagate waves due to singular source terms and treat discontinuous material properties using the multiblock capability of the SBP-SAT approach.

We advance in time using a $6^{\text {th }}$-order Runge-Kutta method [59] and a time step $\Delta t=0.2 \mathrm{~h}$. To measure the error, we define the $l_{2}$-norm $\|e\|_{h}=\left\|U-U^{*}\right\|_{h}$ where $U$ is the numerical solution and $U^{*}$ is computed from a manufactured solution, evaluated at the grid points. The convergence rate is defined as

$$
q=\log _{2}\left(\|e\|_{h_{1}} /\|e\|_{h_{2}}\right)
$$

where $h_{2}=h_{1} / 2$.

In the last two tests below we will estimate errors and convergence rates by comparing to a reference solution computed on the finest grid. Since the staggered grid in Figure 2 is inconvenient for these computations, we will use an alternative staggered grid. This staggered grid places $p$ on the regular grid $\left(x_{+}, y_{+}\right), u$ on $\left(x_{-}, y_{+}\right)$, and $v$ on $\left(x_{+}, y_{-}\right)$. The stability analysis holds for this staggered grid as well because it can be derived from the previously analyzed grid by swapping signs, i.e. $x_{-} \rightarrow x_{+}, x_{+} \rightarrow x_{-}, y_{-} \rightarrow y_{+}$, and $y_{+} \rightarrow y_{-}$. The error can then be estimated as $\left\|p^{(\text {coarse })}-p^{(\text {fine })}\right\|_{h}$, where $p^{(f i n e)}$ is the numerical solution obtained on the finest grid and sampled at the grid points of a coarser grid having solution $p^{(\text {coarse })}$. 


\subsection{Convergence rate}

This test demonstrates that both the collocated and staggered grid SBPSAT schemes converge at the expected theoretical order of accuracy assuming smooth solutions.

Let $\Omega=[0,1] \times[0,1]$ be the computational domain and let

$$
\begin{aligned}
& p(x, y, t)=\sin (k x) \sin (k y) \cos (k \sqrt{2} t), \\
& u(x, y, t)=-\frac{1}{\sqrt{2}} \cos (k x) \sin (k y) \sin (k \sqrt{2} t), \\
& v(x, y, t)=-\frac{1}{\sqrt{2}} \sin (k x) \cos (k y) \sin (k \sqrt{2} t),
\end{aligned}
$$

be the manufactured solution. In this test, we specify the incoming characteristic variable and use the manufactured solution as boundary data. We use $N$ number of grid points in each direction and the wavenumber $k=4 \pi$. Without going into too much detail, the SBP-SAT collocated scheme can be obtained by placing all of the unknowns on the same grid $\left(x_{+}, y_{+}\right)$and by replacing the SBP operators $D_{+}$and $D_{-}$in 28 with the traditional SBP operators $D=P^{-1} Q$, reviewed in [42]. Tables 1,2 list the convergence rate at $t=1$ of the collocated and the staggered scheme, respectively. Both schemes converge at least at the expected theoretical rate of $s+1$, where $2 s$ is the interior accuracy [60]. In several cases, both schemes exceed the ex-

\begin{tabular}{|c|c|c|c|c|c|c|}
\hline \multirow[b]{2}{*}{$N$} & \multicolumn{2}{|l|}{$2^{\text {nd }}$} & \multicolumn{2}{|l|}{$4^{t h}$} & \multicolumn{2}{|l|}{$6^{t h}$} \\
\hline & $\log _{10} l_{2}$-error & $q$ & $\log _{10} l_{2}$-error & $q$ & $\log _{10} l_{2}$-error & $q$ \\
\hline 20 & -0.27 & & -1.18 & & -1.24 & \\
\hline 40 & -0.84 & 1.90 & -2.21 & 3.40 & -2.64 & 4.67 \\
\hline 80 & -1.44 & 1.99 & -3.15 & 3.13 & -3.97 & 4.43 \\
\hline 160 & -2.04 & 2.00 & -4.07 & 3.03 & -5.36 & 4.62 \\
\hline 320 & -2.64 & 2.00 & -4.97 & 3.01 & -6.75 & 4.61 \\
\hline 640 & -3.24 & 2.00 & -5.88 & 3.00 & -8.08 & 4.40 \\
\hline
\end{tabular}
pected theoretical convergence rate. This positive result is, due to a careful optimization of free parameters in the boundary stencils.

Table 1: Relative $\log _{10}$-errors $\|e\|_{h}$ and convergence rates $q$ using the collocated SBP operators. 


\begin{tabular}{|c|c|c|c|c|c|c|}
\hline \multirow[b]{2}{*}{$N$} & \multicolumn{2}{|l|}{$2^{n d}$} & \multicolumn{2}{|l|}{$4^{t h}$} & \multicolumn{2}{|l|}{$6^{t h}$} \\
\hline & $\log _{10} l_{2}$-error & $q$ & $\log _{10} l_{2}$-error & $q$ & $\log _{10} l_{2}$-error & $q$ \\
\hline 20 & -0.69 & & -1.92 & & -2.27 & \\
\hline 40 & -1.40 & 2.37 & -3.42 & 5.00 & -3.73 & 4.86 \\
\hline 80 & -2.03 & 2.09 & -4.66 & 4.10 & -5.22 & 4.93 \\
\hline 160 & -2.64 & 2.02 & -5.84 & 3.92 & -6.58 & 4.53 \\
\hline 320 & -3.24 & 2.01 & -7.02 & 3.94 & -7.94 & 4.51 \\
\hline 640 & -3.84 & 2.00 & -8.22 & 3.97 & -9.29 & 4.51 \\
\hline
\end{tabular}

Table 2: Relative $\log _{10}$-errors $\|e\|_{h}$ and convergence rates $q$ using the staggered SBP operators.

\subsection{Error growth in time}

In this experiment, we investigate the dispersion properties of the scheme. Dispersion errors are most noticeable once the solution has propagated over many wavelengths. This effect can be seen by using periodic boundary conditions. The manufactured solution (37) with $k=4 \pi$ is periodic by construction, and therefore used again. By using Fourier analysis, numerical dispersion relations can easily be derived for the Cauchy problem. The semidiscrete approximation of Cauchy problem uses the same finite difference approximation on a periodic grid. The numerical dispersion relation is derived by inserting $u_{j}(t)=u\left(x_{j}, t\right)=\exp \left(i k x_{j}-\omega t\right)$, into the semi-discrete approximation of the Cauchy problem.

Figure 5.2 shows the numerical dispersion relations for the collocated and staggered scheme for different order of accuracy. Roughly speaking, when $k h$ is small, the numerical solution is well-resolved, whereas when $k h \rightarrow \pi$, it is poorly resolved. The staggered scheme performs much better at large $k h$ compared to the collocated scheme.

We advance in time until the relative error exceeds unity or until $t=1000$. Figure 4 shows the error growth in time. The staggered scheme outperforms the collocated scheme. The difference in accuracy of the staggered scheme compared to the collocated scheme increases with increasing $k h$. This result is in agreement with the dispersion analysis presented in Figure 5.2 .

\subsection{Singular source term}

In this section, we demonstrate that the SBP staggered scheme can accurately propagate waves generated by a singular source term placed on the 


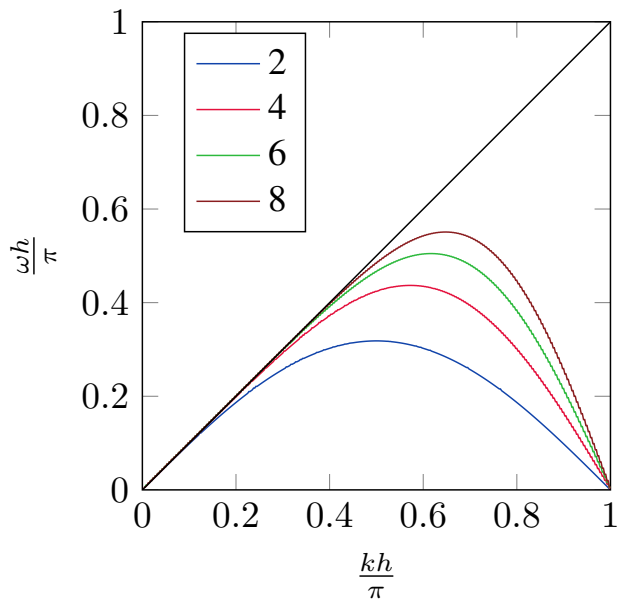

(a) Collocated

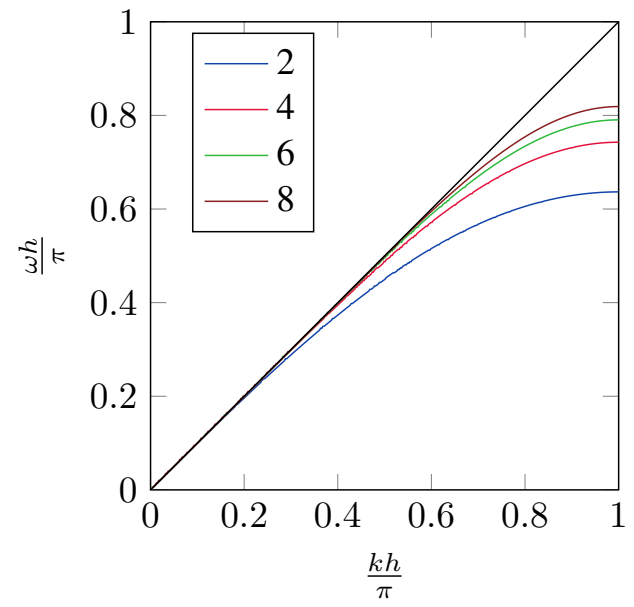

(b) Staggered

Figure 3: Dispersion curves for the collocated and staggered finite difference approximations of the wave equation $p_{t}+u_{x}=0, u_{t}+p_{x}=0$ for $x \in[0,1]$ subject to periodic boundary conditions. The legend states the order of accuracy (second, fourth, ...) of each approximation. The black line is the exact dispersion relation.

boundary.

On the top boundary of the domain $\Omega=[0,2] \times[0,1]$, we specify the boundary condition

$$
p(x, 1, t)=g(t) \delta\left(x-x_{*}\right) .
$$

Here, $\delta\left(x-x_{*}\right)$ is the Dirac delta function, defined as

$$
\int_{-\infty}^{\infty} f(x) \delta\left(x-x_{*}\right) d x=f\left(x_{*}\right),
$$

for some smooth function $f(x)$. We discretize $\delta\left(x-x_{*}\right)$ by using the SBP quadrature rule (3). An approximation of (39) becomes

$$
f^{T} H_{-} d=\mathcal{L}_{*}^{T} f,
$$

where $d$ discretizes the source, and the matrix $\mathcal{L}_{*}$ restricts the grid function $f$ to $x_{*}$. If $x_{*}$ does not coincide with a grid point, then $\mathcal{L}_{*}$ is constructed using an interpolating polynomial of degree $s$ using the $s+1$ nearest grid 


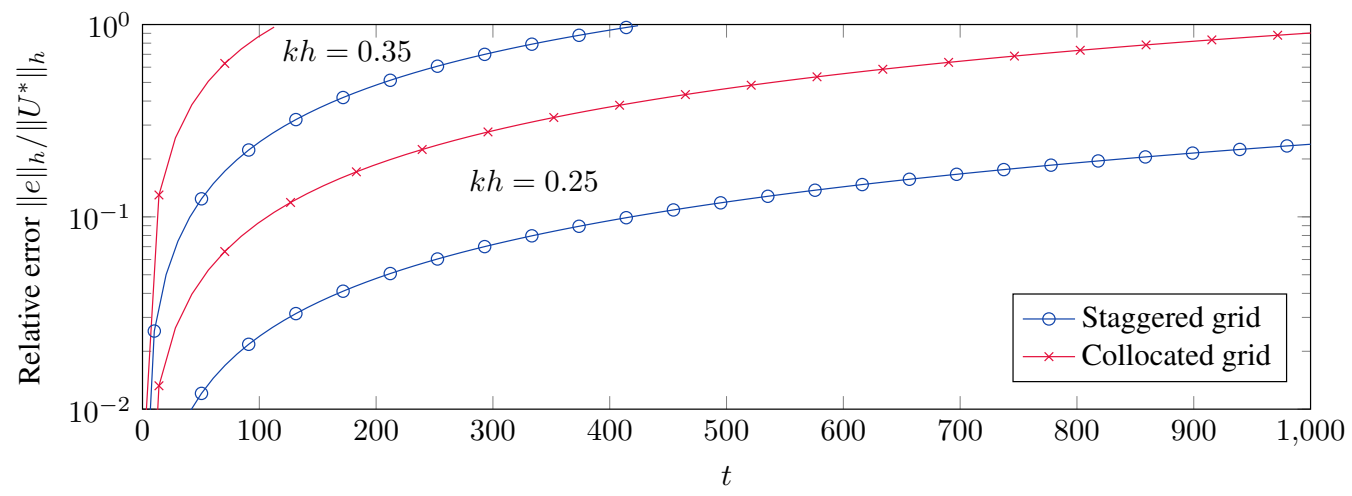

(a) $4^{\text {th }}$-order

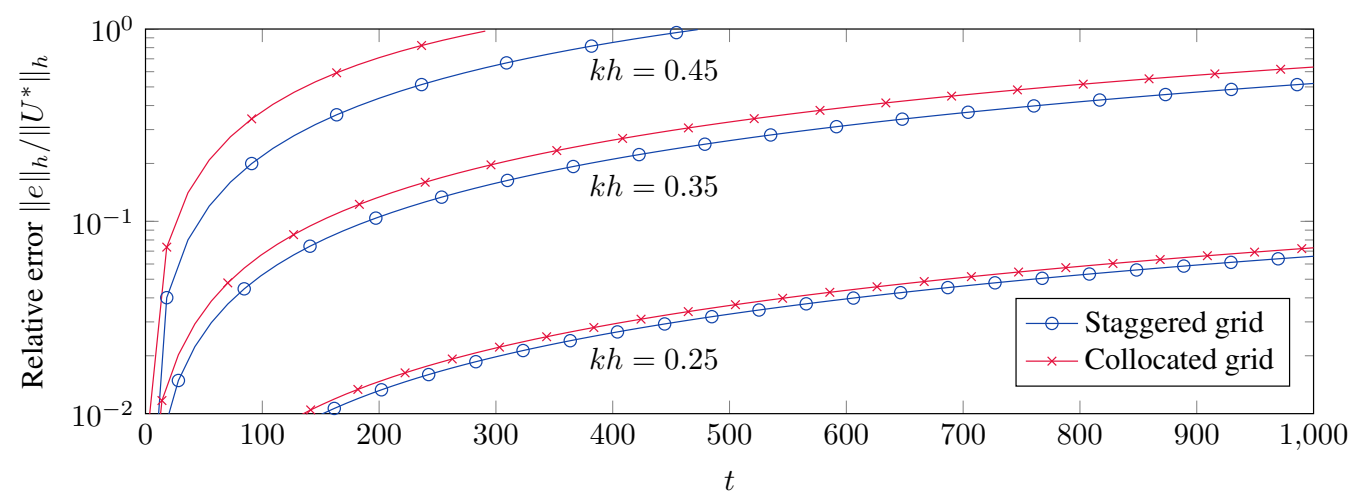

(b) $6^{\text {th }}$-order

Figure 4: Relative error growth in time comparing the staggered and collocated SBP operators for different resolutions $k h$. 
points to the source location. By using monomials $x_{-}^{k}$, we have the moment conditions

$$
d^{T} H_{-} x_{-}^{k}=x_{*}^{k}, k=0,1, \ldots, s .
$$

In the simplest case ( $s=0$ and $x_{*}$ coinciding with a grid point), we have

$$
d_{j+1 / 2}= \begin{cases}\frac{1}{h}, & j=k, \\ 0, & j \neq k .\end{cases}
$$

For collocated schemes that use central difference approximations, the moment conditions (41) are not sufficient for convergence. In this case, spurious oscillations, in the form of large-amplitude highly-oscillatory waves, are generated at the source and propagate throughout the domain. For a solution to this problem, see [61.

For simplicity, we require that the source term location $x_{*}$ coincides with the grid point in the middle of the boundary $\left(x_{*}=1\right)$. As the source time function in (38), we use the Gaussian

$$
g(t)=\exp \left(-\frac{1}{2 a^{2}}\left(t-t_{0}\right)^{2}\right),
$$

where $a=0.015, t_{0}=8 a$. In this test, all fields are initially zero. We discretize the computational domain using the $6^{\text {th }}$-order SBP staggered grid operators and $401 \times 201$ grid points in the $\left(x_{+}, y_{+}\right)$grid, such that $a / h=3$. The parameter $a$ is a representative wavelength (the wave equation is nondimensional) of the source and therefore $a / h$ can be interpreted as the number of grid points per wavelength. Increasing $a / h$ increases the resolution of the numerical solution. Figure 5 shows the pressure wave field at different snapshots in time.

To show high-order convergence, we perform a grid refinement study as explained above. The source location does not coincide with a grid point. Therefore, we need to enforce the moment conditions 41. We use $2 s+1$ moment conditions, where $2 s$ is the interior accuracy. For high-order convergence the solution must be smooth. When the source time function $g(t) \neq 0$, the solution is discontinuous near the source. Either a small region around the source must be removed from the measurement, or a sufficient amount of time $t$ must have passed such that $g(t) \approx 0$. We take the latter approach. Table 3 lists $\log _{10}$-errors and convergence rates. The expected theoretical 


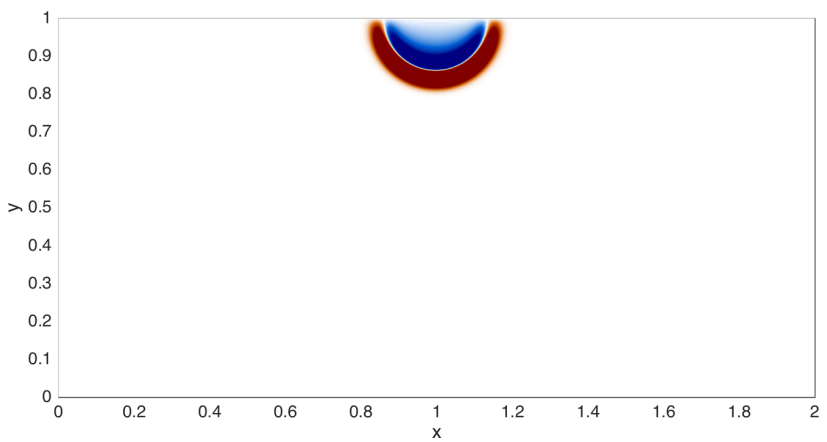

(a) $t=0.3$

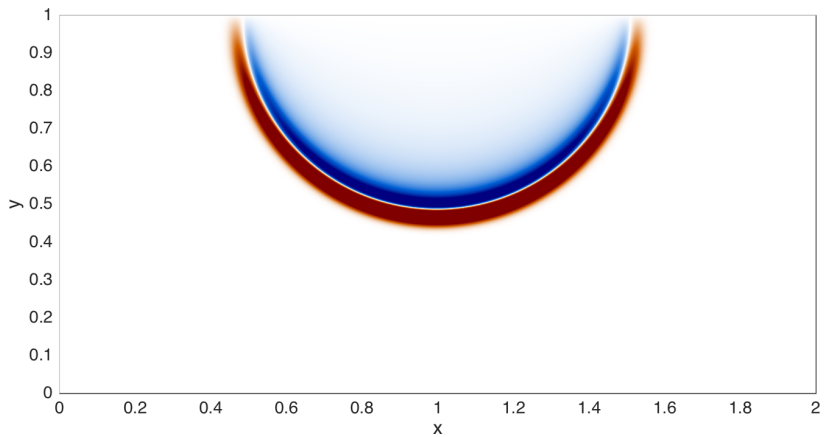

(b) $t=0.6$

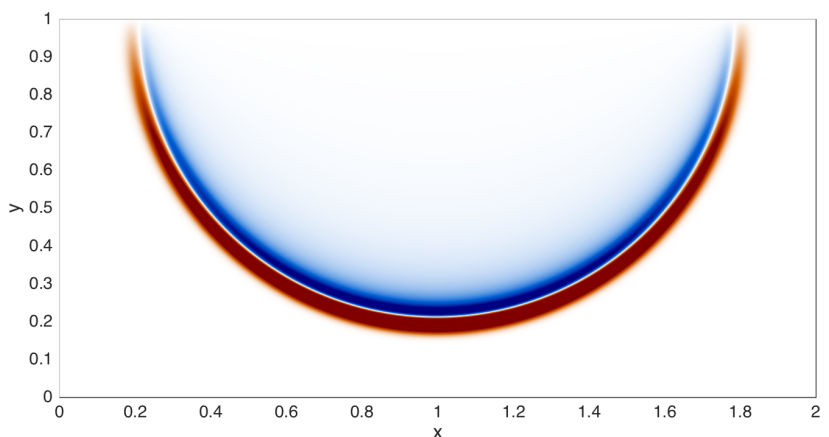

(c) $t=0.9$

Figure 5: Snapshots in time of the pressure field due to a singular source term acting on the boundary. This test uses $400 \times 200$ grid points, $a / h=3$, and the $6^{t h}$-order staggered grid operators. 


\begin{tabular}{|c|c|c|c|c|c|c|}
\hline \multirow[b]{2}{*}{$N$} & \multicolumn{2}{|l|}{$2^{n d}$} & \multicolumn{2}{|l|}{$4^{t h}$} & \multicolumn{2}{|l|}{$6^{t h}$} \\
\hline & $\log _{10} l_{2}$-error & $q$ & $\log _{10} l_{2}$-error & $q$ & $\log _{10} l_{2}$-error & $q$ \\
\hline 50 & -0.50 & & -0.83 & & -0.81 & \\
\hline 100 & -0.89 & 1.29 & -1.69 & 2.86 & -1.91 & 3.64 \\
\hline 200 & -1.51 & 2.07 & -3.03 & 4.43 & -3.75 & 6.10 \\
\hline 400 & -2.13 & 2.05 & -4.30 & 4.22 & -5.48 & 5.75 \\
\hline 800 & -2.73 & 2.01 & -5.50 & 4.00 & -7.20 & 5.72 \\
\hline
\end{tabular}

Table 3: Relative $\log _{10}$-errors $\left\|p^{(\text {coarse })}-p^{(\text {fine })}\right\|_{h}$ and convergence rates $q$ using staggered SBP operators.

convergence rate $s+1$ is again exceeded due to the optimization of free parameters in the boundary stencils. In this convergence study, the following parameters were used: $\Omega=[0,1] \times[0,1], x_{*}=\sqrt{2} / 3, a=0.02, t_{0}=8 a$, and $t=0.5$ such that $g(t) \approx 0$. Note that these parameters are different from the parameters used to produce Figure 5 .

\subsection{Multiblock interface treatment}

In this final experiment, we showcase the multiblock capabilities of the SBP-SAT scheme by coupling subdomains with different material properties. We consider two subdomains located above and below the interface $x=0$. Let $\bar{\Omega}=[0,6] \times[0,1]$ be the top subdomain and let $\Omega=[0,6] \times[-2,0]$ be the bottom subdomain. The wave speed $\bar{c}$ in $\bar{\Omega}$ is lower than the wave speed $c$ in $\Omega$. We use $c=2 \bar{c}=2$ (constant). In each subdomain, we solve the symmetrized, acoustic wave equation,

$$
\begin{aligned}
& \frac{\partial \bar{p}}{\partial t}+\bar{c} \frac{\partial \bar{u}}{\partial x}+\bar{c} \frac{\partial \bar{v}}{\partial y}=0, \quad \frac{\partial \bar{u}}{\partial t}+\bar{c} \frac{\partial \bar{p}}{\partial x}=0, \quad \frac{\partial \bar{v}}{\partial t}+\bar{c} \frac{\partial \bar{p}}{\partial y}=0, \quad(x, y) \in \bar{\Omega}, t \geq 0 \\
& \frac{\partial p}{\partial t}+c \frac{\partial u}{\partial x}+c \frac{\partial v}{\partial y}=0, \quad \frac{\partial u}{\partial t}+c \frac{\partial p}{\partial x}=0, \quad \frac{\partial v}{\partial t}+c \frac{\partial p}{\partial y}=0, \quad(x, y) \in \Omega, t \geq 0
\end{aligned}
$$

Since the wave speed is discontinuous across the interface, we discretize $\Omega$ and $\bar{\Omega}$ using a multiblock discretization. The discretization is grid conforming, i.e., the grid points of each block along the interface are collocated. The two subdomains are coupled at the interface by enforcing

$$
\tilde{p}(0, y, t)=\tilde{\bar{p}}(0, y, t), \tilde{u}(0, y, t)=\tilde{\bar{u}}(0, y, t),
$$


see Appendix A for details on the SBP-SAT implementation. On the top boundary, we impose the free-surface boundary condition

$$
p(x, 1, t)=0 .
$$

All fields are initialized to zero. To excite waves, we add the following singular source term to 44 ,

$$
\frac{\partial \bar{p}}{\partial t}+\bar{c} \frac{\partial \bar{u}}{\partial x}+\bar{c} \frac{\partial \bar{v}}{\partial y}=\delta\left(x-x_{*}\right) \delta\left(y-y_{*}\right) g(t) .
$$

The source location is taken to be $\left(x_{*}=3, y_{*}=0.5\right)$ (located in the center of $\bar{\Omega})$. The source time function is given by the Gaussian function

$$
g(t)=\exp \left(-\frac{1}{2 a^{2}}\left(t-t_{0}\right)^{2}\right),
$$

with $a=0.125$ and $t_{0}=6 a$. We introduce the following parameters to establish how well-resolved the simulation is in each direction (these parameters can be interpreted as the number of grid points per wavelength)

$$
\begin{aligned}
& \bar{M}_{x}=\frac{a \bar{c}}{\bar{h}_{x}} \quad \text { and } \quad \bar{M}_{y}=\frac{a \bar{c}}{\bar{h}_{y}}, \quad(x, y) \in \bar{\Omega}, \\
& M_{x}=\frac{a c}{h_{x}} \quad \text { and } \quad M_{y}=\frac{a c}{h_{y}}, \quad(x, y) \in \bar{\Omega},
\end{aligned}
$$

where $\bar{h}$ and $h$ is the grid spacing in each subdomain, respectively.

In our first test, we use $M_{x}=M_{y}=12$ and $\bar{M}_{x}=\bar{M}_{y}=6$, i.e., the grid spacing is uniform $\left(h_{x}=h_{y}=\bar{h}_{x}=\bar{h}_{y}\right)$. Figure 6 shows snapshots in time of the pressure field. In this simulation, waves from an explosion are reflected against the free surface and material interface. We have also solved this problem using a lower resolution $\bar{M}=3$ and $M=6$. Both simulations are in excellent agreement (cf. Figure 6c and Figure 7a). Table 4 lists relative $\log _{10}$-errors and convergence rates. The expected theoretical convergence rate $s+1$ is again exceeded due to optimization. In this convergence study, the following parameter were used: $\bar{\Omega}=[0,1] \times[0,0.5], \bar{\Omega}=[0,1] \times[0.5,1]$, $h_{x}=h_{y}=\bar{h}_{x}=\bar{h}_{y},\left(x_{*}, y_{*}\right)=(0.5,0.6), a=0.02, t_{0}=8 a$, and $t=0.5$. Note that these parameters are different from the parameters used to produce Figure 6.

We also compare the multiblock approach to a single-block-discretization. The single-block-discretization inaccurate due to differentiation across the 


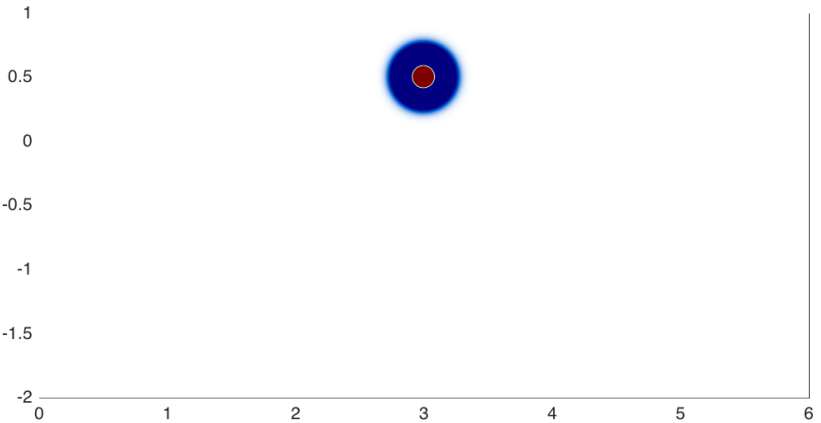

(a) $t=1$

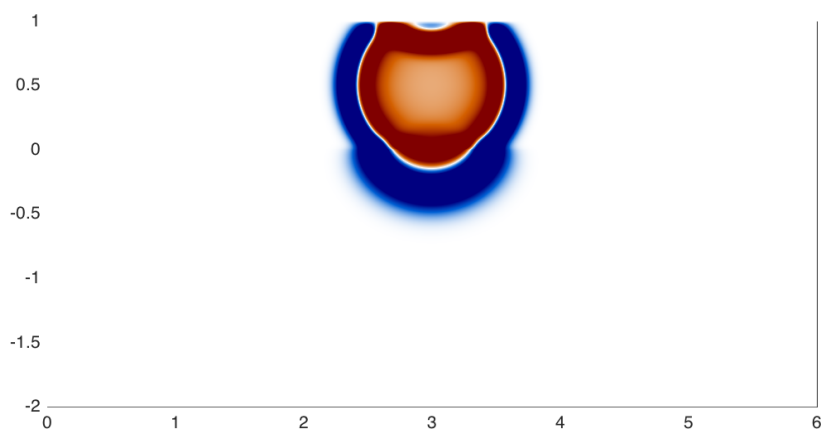

(b) $t=2$

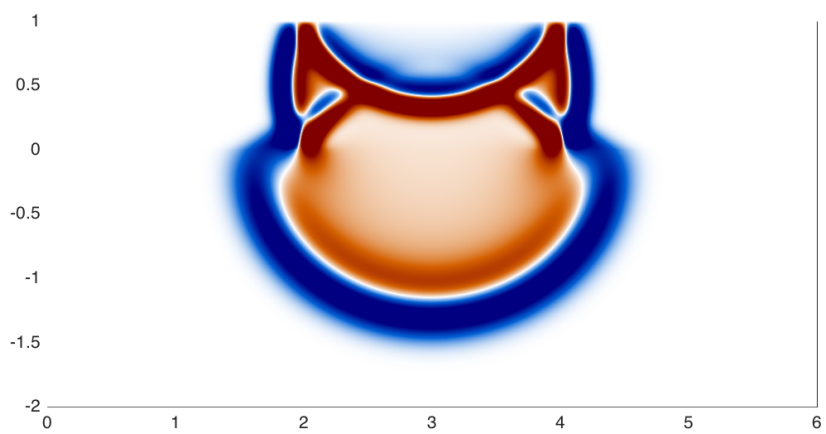

(c) $t=3$

Figure 6: Snapshots in time of the pressure field. This test uses $4^{t h}$-order SBP staggered grid operators on a multiblock grid. The grid resolution is $\bar{M}=6$ and $M=12(768 \times 384$ grid points). 


\begin{tabular}{ccccccccc}
\hline & \multicolumn{2}{c}{$2^{\text {nd }}$} & & & $4^{\text {th }}$ & & \multicolumn{2}{l}{$6^{\text {th }}$} \\
\cline { 2 - 3 }$N_{x}$ & $\log _{10} l_{2}$-error & $q$ & & $\log _{10} l_{2}$-error & $q$ & & $\log _{10} l_{2}$-error & $q$ \\
\hline 50 & -0.37 & & & -0.79 & & & -0.98 & \\
100 & -0.82 & 1.50 & & -1.79 & 3.32 & & -2.28 & 4.31 \\
200 & -1.42 & 1.98 & & -2.99 & 3.97 & & -3.85 & 5.20 \\
400 & -2.03 & 2.03 & & -4.19 & 4.00 & & -5.60 & 5.83 \\
800 & -2.63 & 2.01 & & -5.40 & 4.00 & & -7.40 & 5.98 \\
\hline
\end{tabular}

Table 4: Relative $\log _{10}$-errors and convergence rates $q$ using the SBP staggered grid operators.

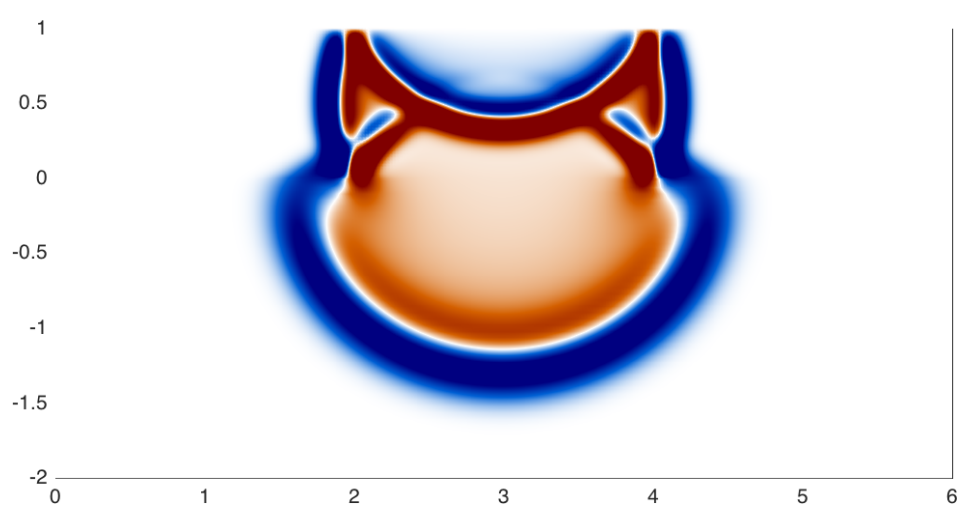

(a) $\bar{M}_{x}=3, \bar{M}_{y}=3$ and $M_{x}=6, M_{y}=3$

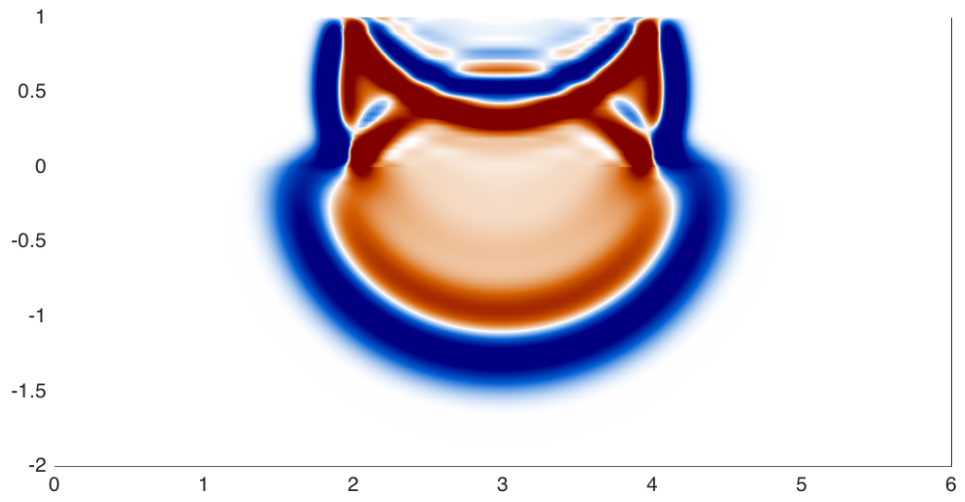

(b) $\bar{M}_{x}=3, \bar{M}_{y}=1.5$ and $M_{x}=6, M_{y}=3$

Figure 7: The pressure at time $t=3$ in $\bar{\Omega}$ (a) accurate resolution with constant resolution in the $y$-direction. (b) Inaccurate result due to under-resolving the solution in the $y$ direction 
material discontinuity. There is another disadvantage with the single-blockdiscretization. The subdomain $\Omega$ is over-resolved because the grid resolution has been set by the accuracy requirements of $\bar{\Omega}$, having the shortest wavelength. With the multiblock approach, we increase the grid spacing in the $y$-direction of $\Omega$ such that we have the same resolution $\bar{M}_{y}=M_{y}$ in each subdomain. In this test, the modification reduces the computational cost by $35 \%$ without destroying the accuracy of the solution (Figure 7 a). To confirm that we have not over-resolved the solution, we also increase the grid spacing in the $y$-direction in $\bar{\Omega}$. As expected, Figure $7 \mathrm{~b}$ shows that this modification results in visible errors.

One can obtain a more significant improvement in computational efficiency by also coarsening the mesh in the x-direction, but this requires using non-conforming grids. Many methods have been proposed for interpolating across non-conforming, staggered grids, see e.g., [62, 63, 64] and the references therein. To the best of our knowledge, these methods are restricted to coarse-to-fine grid spacing ratios of integer order and have no proofs of stability. With SBP-SAT, provably stable interpolation methods have been developed for non-conforming, collocated grids [65, 50]. These methods should be directly applicable or straightforward to extend to staggered grids.

\section{Conclusions}

In this work, we extended the SBP-SAT approach to staggered grids. This extension combines the excellent dispersion properties of high-order accurate finite difference approximations on staggered grids with the excellent stability properties of the SBP-SAT approach. The key idea that made this extension possible was a new summation-by-parts property. This property, together with a weak enforcement of boundary conditions, enabled us to show stability by deriving a discrete energy estimate. Although we only considered the scalar wave equation, the method can likely be applied to the elastic wave equation, Maxwell's equations, and various anisotropic scalar wave equations with minor modifications. While we only considered homogeneous boundary conditions, it is straightforward to apply the method to problems with more complex boundary or interface conditions, such as, for example, those arising when modeling earthquakes and tsunamis [34, 35, 36, 37], etc.

To assess the accuracy and showcase the capabilities of the method, we performed a series of numerical experiments. In all of the numerical 
experiments, high-order convergence was demonstrated using either a manufactured solution or by computing a reference solution on a fine grid. First, a standing wave solution was used to investigate the accuracy of SBP staggered grid and collocated grid operators. The SBP staggered grid operators outperformed the SBP collocated grid operators. Furthermore, the convergence rate of the SBP staggered grid operators exceeded the expected theoretical convergence rate of $s+1$. Second, we ran a test with a singular source term on the boundary and showed high-order convergence once the source time function was no longer active.

Finally, we applied an SBP-SAT multiblock discretization to obtain a high-order-accurate solution to a problem with a material discontinuity. We improved the computational cost compared to a single block discretization by reducing the number of grid points in the normal direction with respect to the interface of the block with the largest wave speeds. The multiblock capability of SBP-SAT schemes is a powerful tool that extends beyond the discretization of discontinuous material structures. For example, it can be used to couple non-conforming grids, unstructured and structured grids, as well as to solve coupled multi-physics problems.

\section{7. * Acknowledgments}

O. O'Reilly was partially supported by the Chevron fellowship in the Department of Geophysics at Stanford University.

\section{Appendix A. Multiblock coupling}

In this section, we present a stable, multiblock coupling procedure using SBP-SAT. We only consider the one dimensional case, but the analysis can be extended to two dimensions by following the procedure laid out in Section 4.2 . Without loss of generality, we couple the left boundary to the right boundary of a single block (the multiblock coupling uses the same conditions). Consider the unsymmetrized wave equation with variable coefficients:

$$
\frac{1}{K} \frac{\partial \tilde{p}}{\partial t}+\frac{\partial \tilde{u}}{\partial x}=0, \rho \frac{\partial \tilde{u}}{\partial t}+\frac{\partial \tilde{p}}{\partial x}=0 .
$$

where $K=K(x)$ is the bulk modulus, $\rho=\rho(x)$ is the density, and $c(x)=$ $\sqrt{K / \rho}$ is the wave speed. The mechanical energy per unit area is

$$
E=\frac{1}{2} \int_{0}^{1} \frac{\tilde{p}^{2}}{K}+\rho \tilde{u}^{2} d x
$$


where the first term is the elastic energy and the second term is the kinetic energy. By taking the time derivative of A.2 and using A.1 , we obtain

$$
\frac{d E}{d t}=-\left.\tilde{p} \tilde{u}\right|_{0} ^{1}
$$

The appropriate physical coupling conditions are obtained by requiring the pressure and normal velocity to be continuous at the interface:

$$
\left.\tilde{p}\right|_{0}=\left.\tilde{p}\right|_{1} \quad \text { and }\left.\quad \tilde{u}\right|_{0}=\left.\tilde{u}\right|_{1} .
$$

A semi-discrete approximation of $(\mathrm{A} .4)$ is written as

$$
\begin{array}{r}
K^{-1} \frac{d \tilde{p}}{d t}+D_{-} \tilde{u}=P_{-}^{-1} e_{N-}\left(\tilde{u}_{N}-\tilde{u}^{*}\right)-P_{-}^{-1} e_{0-}\left(\tilde{u}_{0}-\tilde{u}^{*}\right), \\
\rho \frac{d \tilde{u}}{d t}+D_{-} \tilde{p}=P_{+}^{-1} e_{N+}\left(\tilde{p}_{N}-\tilde{p}^{*}\right)-P_{+}^{-1} e_{0+}\left(\tilde{p}_{0}-\tilde{p}^{*}\right),
\end{array}
$$

where $K=\operatorname{diag}\left(\left[K_{0}, K_{1 / 2}, \ldots, K_{N-1 / 2}, K_{N}\right]\right)$ and $\rho=\operatorname{diag}\left(\left[\rho_{0}, \rho_{1}, \ldots, \rho_{N}\right]\right)$ hold the material properties at each grid point. The coupling conditions A.3 are enforced by choosing the numerical fluxes $\tilde{p}^{*}$ and $\tilde{u}^{*}$. We use

$$
\tilde{p}^{*}=\frac{\alpha \beta}{\alpha+\beta}\left(\tilde{u}_{N}-\tilde{u}_{0}\right)+\frac{\beta \tilde{p}_{0}+\alpha \tilde{p}_{N}}{\alpha+\beta} \text { and } \quad \tilde{u}^{*}=\frac{\alpha \tilde{u}_{0}+\beta \tilde{u}_{N}}{\alpha+\beta}+\frac{\tilde{p}_{N}-\tilde{p}_{0}}{\alpha+\beta} .
$$

where $\alpha$ and $\beta$ are the acoustic impedance of the medium on each side of the interface $\left(\alpha=\rho_{0} c_{0}>0\right.$ and $\left.\beta=\rho_{N} c_{N}>0\right)$. This choice takes into account that the material properties $\rho$ and $K$ can be discontinuous across the interface. Except for a specific choice of $\alpha$ and $\beta$, the numerical fluxes A.5) introduce energy dissipation (see Proposition 3 below), which may be undesirable. A fully energy conservative coupling is obtained by choosing

$$
\tilde{p}^{*}=\tilde{p}_{0} \quad \text { and } \quad \tilde{u}^{*}=\tilde{u}_{N},
$$

or

$$
\tilde{p}^{*}=\tilde{p}_{N} \quad \text { and } \quad \tilde{u}^{*}=\tilde{u}_{0} .
$$

In each case above, the coupling conditions A.3 are enforced in a staggered manner, and each specific choice of the numerical fluxes arise as a special case of the general formulation (A.5) (e.g., choose $\alpha=1 / \beta=\xi$ in (A.5), and take the limit $\xi \rightarrow 0$ to obtain (A.6)). By using the SBP property (4), we can easily show stability. We have the following proposition. 
Proposition 3. The semi-discrete approximation (A.4) of (A.1) using the numerical fluxes $(A .5)$ is stable.

Proof. Analogous to the continuous energy A.2, a discrete energy is defined as

$$
E_{h}=\frac{1}{2} \tilde{p}^{T} K^{-1} P_{-} \tilde{p}+\frac{1}{2} \tilde{u}^{T} \rho P_{+} \tilde{u}
$$

By multiplying each equation in A.4 by $\tilde{p}^{T} P_{-}$and $\tilde{u}^{T} P_{+}$from the left, and by using the SBP property (4), we get

$$
\begin{aligned}
\frac{d E_{h}}{d t}= & -\tilde{p}^{T}\left(Q_{+}+Q_{-}^{T}\right) \tilde{u}+\tilde{p}_{N}\left(\tilde{u}_{N}-\tilde{u}^{*}\right)-\tilde{p}_{0}\left(\tilde{u}_{0}-\tilde{u}^{*}\right) \\
& +\tilde{u}_{N}\left(\tilde{p}_{N}-\tilde{p}^{*}\right)-\tilde{u}_{0}\left(\tilde{p}_{0}-\tilde{p}^{*}\right) \\
= & \tilde{p}_{N} \tilde{u}_{N}-\tilde{p}_{0} \tilde{u}_{0}+\left(\tilde{p}_{0}-\tilde{p}_{N}\right) \tilde{u}^{*}+\left(\tilde{u}_{0}-\tilde{u}_{N}\right) \tilde{p}^{*} .
\end{aligned}
$$

Finally, by inserting the numerical fluxes A.5 into A.9), we get

$$
\frac{d E_{h}}{d t}=-\frac{1}{\alpha+\beta}\left(\tilde{p}_{0}-\tilde{p}_{N}\right)^{2}-\frac{\alpha \beta}{\alpha+\beta}\left(\tilde{u}_{0}-\tilde{u}_{N}\right)^{2} \leq 0 .
$$

The result A.10 shows that numerical dissipation is introduced in the scheme if $\alpha+\beta>0$, and this dissipation vanishes with grid refinement as the numerical solution converges to the solution of the continuous problem. In the limit $\xi \rightarrow 0$ where $\xi=\alpha=1 / \beta$, or $\xi=\beta=1 / \alpha$, the energy in A.10 is conserved. In this case, the numerical fluxes are chosen as (A.6), or (A.7).

[1] K. S. Yee, et al., Numerical solution of initial boundary value problems involving maxwells equations in isotropic media, IEEE Trans. Antennas Propag 14 (3) (1966) 302-307. doi:10.1109/tap.1966.1138693. URL http://dx.doi.org/10.1109/tap.1966.1138693

[2] J. Fang, Time domain finite difference computation for maxwell's equations, Ph.D. thesis, University of California, Berkeley (1989).

[3] C. M. Jr, S. L. Broschat, J. B. Schneider, Higher-order fdtd methods for large problems, J. Applied Computational Electromagnetics Society 10 (1995) 17-29. 
[4] E. Turkel, A. Yefet, Fourth order method for maxwell equations on a staggered mesh, in: IEEE Antennas and Propagation Society International Symposium 1997. Digest, Vol. 4, 1997, pp. 2156-2159 vol.4. doi:10.1109/APS.1997.625395.

[5] A. Yefet, E. Turkel, Fourth order compact implicit method for the Maxwell equations with discontinuous coefficients, Applied Numerical Mathematics 33 (1) (2000) 125-134. doi:10.1016/S0168-9274(99) 00075-6.

[6] A. Taflove, S. C. Hagness, Computational Electrodynamics: The FiniteDifference Time-Domain Method, Third Edition (2005). doi:10.1515/ 9783110809824.v.

[7] J. Virieux, SH-wave propagation in heterogeneous media: Velocity-stress finite-difference method, Geophys. 49 (11) (1984) 1933-1942. doi:10. $1190 / 1.1441605$.

URL http://dx.doi.org/10.1190/1.1441605

[8] J. Virieux, P-SVwave propagation in heterogeneous media: Velocitystress finite-difference method, Geophys. 51 (4) (1986) 889-901. doi: 10.1190/1.1442147.

URL http://dx.doi.org/10.1190/1.1442147

[9] A. R. Levander, Fourth-order finite-differenceP-SVseismograms, Geophys. 53 (11) (1988) 1425-1436. doi:10.1190/1.1442422.

URL http://dx.doi.org/10.1190/1.1442422

[10] R. W. Graves, Simulating seismic wave propagation in 3d elastic media using staggered-grid finite differences, Bull. Seism. Soc. Am. 86 (4) (1996) 1091-1106.

[11] H.-O. Kreiss, J. Oliger, Comparison of accurate methods for the integration of hyperbolic equations, Tellus XXIV (1972) 199-215.

[12] B. Fornberg, High-Order Finite Differences and the Pseudospectral Method on Staggered Grids, SIAM Journal on Numerical Analysis 27 (4) (1990) 904-918. doi:10.1137/0727052.

URL http://dx.doi.org/10.1137/0727052 
[13] D. Gottlieb, B. Yang, Comparisons of staggered and non-staggered schemes for maxwells equations, in: 12th Annual Review of Progress in Applied Computational Electromagnetics, Vol. 2, 1996, pp. 1122-1131.

[14] D. W. Zingg, Comparison of High-Accuracy Finite-Difference Methods for Linear Wave Propagation, SIAM Journal on Scientific Computing 22 (2) (2000) 476-502. doi:10.1137/S1064827599350320.

[15] M. Ghrist, B. Fornberg, T. A. Driscoll, Staggered time integrators for wave equations, SIAM J. Numer. Anal. 38 (3) (2000) 718-741. doi: $10.1137 / \mathrm{s} 0036142999351777$.

URL http://dx.doi.org/10.1137/s0036142999351777

[16] P. Monk, E. Süli, Error Estimates for Yee's Method on Non-uniform Grids, IEEE Transactions on Magnetics 30 (5) (1994) 3200-3203. doi: $10.1109 / 20.312618$.

[17] A. Ditkowski, K. Dridi, J. Hesthaven, Convergent Cartesian Grid Methods for Maxwell's Equations in Complex Geometries, Journal of Computational Physics 170 (1) (2001) 39-80. doi:10.1006/jcph.2001.6719. URL http://linkinghub.elsevier.com/retrieve/pii/ S0021999101967191]

[18] S. Abarbanel, A. Ditkowski, A. Yefet, Bounded error schemes for the wave equation on complex domains, Journal of Scientific Computing 26 (1) (2006) 67-81. doi:10.1007/s10915-004-4800-x.

[19] J. Häggblad, O. Runborg, Accuracy of staircase approximations in finitedifference methods for wave propagation, Numer. Math 128 (4) (2014) 741-771. doi:10.1007/s00211-014-0625-1.

URL http://dx.doi.org/10.1007/s00211-014-0625-1

[20] A. Yefet, P. G. Petropoulos, A Staggered Fourth-Order Accurate Explicit Finite Difference Scheme for the Time-Domain Maxwell's Equations, Journal of Computational Physics 168 (2) (2001) 286-315. doi:10.1006/jcph.2001.6691.

URL http://www.sciencedirect.com/science/article/pii/ S0021999101966914 
[21] Z. Xie, C.-H. Chan, B. Zhang, An Explicit Fourth-Order Orthogonal Curvilinear Staggered-Grid FDTD Method for Maxwell's Equations, Journal of Computational Physics 175 (2) (2002) 739-763. doi:10.1006/jcph.2001.6965.

URL http://linkinghub.elsevier.com/retrieve/pii/ S0021999101969657

[22] S. Hestholm, B. Ruud, 2D finite difference elastic wave modeling including surface topography, Geophysical Prospecting 42 (November 1992) (1994) 371-390.

[23] I. Tarrass, L. Giraud, P. Thore, New curvilinear scheme for elastic wave propagation in presence of curved topography, Geophysical Prospecting 59 (5) (2011) 889-906. doi:10.1111/j.1365-2478.2011.00972.x.

[24] C. A. Pérez Solano, D. Donno, H. Chauris, Finite-difference strategy for elastic wave modelling on curved staggered grids, Computational Geosciences 20 (1) (2016) 245-264. doi:10.1007/s10596-016-9561-8.

[25] C. Zhang, R. J. LeVeque, The immersed interface method for acoustic wave equations with discontinuous coefficients, Wave Motion 25 (3) (1997) 237-263. doi:http://dx.doi.org/10.1016/S0165-2125(97) 00046-2.

URL http://www.sciencedirect.com/science/article/pii/ S0165212597000462

[26] T. a. Driscoll, B. Fornberg, A Block Pseudospectral Method for Maxwell's Equations, Journal of Computational Physics 65 (1998) 47-65. doi:10.1006/jcph.1998.5883.

URL http://citeseerx.ist.psu.edu/viewdoc/download?doi=10. $1.1 .15 .8997\{\&\}$ rep $=$ rep $1\{\&\}$ type $=$ pdf

[27] J. Hesthaven, High-order accurate methods in time-domain computational electromagnetics: A review, in: Advances in Imaging and Electron Physics, Elsevier BV, 2003, pp. 59-123. doi:10.1016/s1076-5670(03) 80097-6.

URL http://dx.doi.org/10.1016/s1076-5670(03)80097-6

[28] T. Xiao, Q. H. Liu, A staggered upwind embedded boundary (SUEB) method to eliminate the FDTD staircasing error, IEEE Transactions on 
Antennas and Propagation 52 (3) (2004) 730-741. doi:10.1109/TAP. 2004.824675.

[29] S. Zhao, G. W. Wei, High-order FDTD methods via derivative matching for Maxwell's equations with material interfaces, Journal of Computational Physics 200 (1) (2004) 60-103. doi:10.1016/j.jcp.2004.03. 008 .

[30] D. D. Nguyen, S. Zhao, A second order dispersive FDTD algorithm for transverse electric Maxwell's equations with complex interfaces, Computers and Mathematics with Applications 71 (4) (2015) 1010-1035. doi:10.1016/j. camwa.2016.01.014.

URL http://dx.doi.org/10.1016/j. camwa.2016.01.014

[31] T. Rylander, A. Bondeson, Stable FEM-FDTD hybrid method for Maxwell 's equations, Computer Physics Communications 125 (2000) 75-82. doi:10.1016/S0010-4655(99)00463-4.

[32] A. Monorchio, A. Rubio Bretones, R. Mittra, G. Manara, R. Gómez Martín, A hybrid time-domain technique that combines the finite element, finite difference and method of moment techniques to solve complex electromagnetic problems, IEEE Transactions on Antennas and Propagation 52 (10) (2004) 2666-2674. doi:10.1109/TAP.2004. 834431 .

[33] G. C. Lotto, E. M. Dunham, High-order finite difference modeling of tsunami generation in a compressible ocean from offshore earthquakes, Computational Geosciences 19 (2) (2015) 327-340.

[34] J. E. Kozdon, E. M. Dunham, J. Nordström, Interaction of waves with frictional interfaces using summation-by-parts difference operators: Weak enforcement of nonlinear boundary conditions, Journal of Scientific Computing 50 (2012) 341-367. doi:10.1007/s10915-011-9485-3.

[35] J. E. Kozdon, E. M. Dunham, J. Nordström, Simulation of dynamic earthquake ruptures in complex geometries using high-order finite difference methods, J. Sci. Comput. 55 (1) (2013) 92-124. doi:10.1007/ s10915-012-9624-5.

[36] O. O'Reilly, J. Nordström, J. E. Kozdon, E. M. Dunham, Simulation of earthquake rupture dynamics in complex geometries using coupled finite 
difference and finite volume methods, J. Commun. Phys. 17 (02) (2015)

337-370. doi:10.4208/cicp.111013.120914a.

URL http://dx.doi.org/10.4208/cicp.111013.120914a

[37] K. Duru, E. M. Dunham, Dynamic earthquake rupture simulations on nonplanar faults embedded in 3d geometrically complex, heterogeneous elastic solids, Journal of Computational Physics 305 (2016) 185-207.

[38] Y. Zhang, D. D. Nguyen, K. Du, J. Xu, S. Zhao, Time-domain numerical solutions of maxwell interface problems with discontinuous electromagnetic time-domain numerical solutions of maxwell interface problems with discontinuous electromagnetic waves, Advances in Applied Mathematics and Mechanics 8 (3) (2016) 353-385. doi:10.4208/aamm. 2014. m811.

[39] H. Kreiss, G. Scherer, Finite element and finite difference methods for hyperbolic partial differential equations, Academic Press, 1974. doi: 10.1016/b978-0-12-208350-1.50012-1.

[40] B. Strand, Summation by parts for finite difference approximations for d/dx, J. Comput. Phys. 110 (1) (1994) 47-67. doi:10.1006/jcph. 1994.1005.

URL http://dx.doi.org/10.1006/jcph.1994.1005

[41] P. Olsson, Summation by parts, projections, and stability, Math. Comput. 64 (1995) 1035-1035. doi:10.2307/2153482.

[42] M. Svärd, J. Nordström, Review of summation-by-parts schemes for initial-boundary-value problems, J. Comput. Phys. 268 (2014) 17-38. doi:10.1016/j.jcp.2014.02.031.

URL http://dx.doi.org/10.1016/j.jcp.2014.02.031

[43] M. H. Carpenter, D. Gottlieb, Spectral Methods on Arbitrary Grids, Journal of Computational Physics 129 (1) (1996) 74-86. doi:http://dx.doi.org/10.1006/jcph.1996.0234.

URL http://wwW.sciencedirect.com/science/article/pii/ S002199919690234X

[44] G. J. Gassner, A skew-symmetric discontinuous galerkin spectral element discretization and its relation to sbp-sat finite difference methods, SIAM Journal on Scientific Computing 35 (3) (2013) A1233-A1253. 
[45] H. Ranocha, P. Öffner, T. Sonar, Summation-by-parts operators for correction procedure via reconstruction, Journal of Computational Physics 311 (2016) 299-328.

[46] J. E. Hicken, D. W. Zingg, Superconvergent functional estimates from summation-by-parts finite-difference discretizations, SIAM J. Sci. Comput. 33 (2) (2011) 893-922. doi:10.1137/100790987. URL http://dx.doi.org/10.1137/100790987

[47] J. Berg, J. Nordström, On the impact of boundary conditions on dual consistent finite difference discretizations, J. Comput. Phys. 236 (2013) 41-55. doi:10.1016/j.jcp.2012.11.019. URL http://dx.doi.org/10.1016/j.jcp.2012.11.019

[48] J. Nordström, J. Gong, A stable and efficient hybrid method for aeroacoustic sound generation and propagation, C R Mecanique 333 (9) (2005) 713-718. doi:10.1016/j.crme.2005.07.011. URL http://dx.doi.org/10.1016/j.crme.2005.07.011

[49] J. Nordström, J. Gong, A stable hybrid method for hyperbolic problems, J. Comput. Phys. 212 (2) (2006) 436-453. doi:10.1016/j.jcp. 2005. 07.008 .

URL http://dx.doi.org/10.1016/j.jcp.2005.07.008

[50] J. E. Kozdon, L. C. Wilcox, Stable coupling of nonconforming, highorder finite difference methods, SIAM J. Sci. Comput. 38 (2) (2016) A923-A952. doi:10.1137/15m1022823.

URL http://dx.doi.org/10.1137/15m1022823

[51] S. Patankar, Numerical heat transfer and fluid flow, CRC press, 1980.

[52] A. Meurer, C. P. Smith, M. Paprocki, O. Čertík, S. B. Kirpichev, M. Rocklin, A. Kumar, S. Ivanov, J. K. Moore, S. Singh, T. Rathnayake, S. Vig, B. E. Granger, R. P. Muller, F. Bonazzi, H. Gupta, S. Vats, F. Johansson, F. Pedregosa, M. J. Curry, A. R. Terrel, v. Roučka, A. Saboo, I. Fernando, S. Kulal, R. Cimrman, A. Scopatz, Sympy: symbolic computing in python, PeerJ Computer Science 3 (2017) e103. doi:10.7717/peerj-cs.103.

URL https://doi.org/10.7717/peerj-cs.103 
[53] K. Mattsson, M. Almquist, M. H. Carpenter, Optimal diagonal-norm SBP operators, Journal of Computational Physics 264 (2014) 91-111. doi:10.1016/j.jcp.2013.12.041.

URL http://dx.doi.org/10.1016/j.jcp. 2013.12.041

[54] B. Gustafsson, H.-O. Kreiss, J. Oliger, Time-Dependent Problems and Difference Methods, Wiley-Blackwell, 2013. doi:10.1002/ 9781118548448 .

URL http://dx.doi.org/10.1002/9781118548448

[55] S. V. Tsynkov, Numerical solution of problems on unbounded domains. A review, Applied Numerical Mathematics 27 (4) (1998) 465-532. doi: 10.1016/S0168-9274(98)00025-7.

[56] D. Givoli, High-order local non-reflecting boundary conditions: A review, Wave Motion 39 (4) (2004) 319-326. doi:10.1016/j.wavemoti. 2003.12 .004

[57] J. P. Berenger, A perfectly matched layer for the absorption of electromagnetic waves, J. Comput. Phys. 114 (1994) 185-200. arXiv: 0021-9991, doi:10.1006/jcph.1994.1159.

URL http://portal.acm .org/citation.cfm?id=195266

[58] D. Appelö, T. Colonius, A high-order super-grid-scale absorbing layer and its application to linear hyperbolic systems, Journal of Computational Physics 228 (11) (2009) 4200-4217. doi:http://dx.doi.org/10.1016/j.jcp.2009.02.030

URL http://www.sciencedirect.com/science/article/pii/ S0021999109001089

[59] E. A. Alshina, E. M. Zaks, N. N. Kalitkin, Optimal first- to sixth-order accurate runge-kutta schemes, Computational Mathematics and Mathematical Physics 48 (3) (2008) 395-405. doi:10.1134/ S0965542508030068.

URL http://dx.doi.org/10.1134/S0965542508030068

[60] M. Svärd, J. Nordström, On the order of accuracy for difference approximations of initial-boundary value problems, J. Comput. Phys. 218 (1) (2006) 333-352. doi:10.1016/j.jcp. 2006.02.014.

URL http://dx.doi.org/10.1016/j.jcp.2006.02.014 
[61] N. A. Petersson, O. O’Reilly, B. Sjögreen, S. Bydlon, Discretizing singular point sources in hyperbolic wave propagation problems, J. Comput. Phys. 321 (2016) 532-555. doi:10.1016/j.jcp.2016.05.060.

URL http://dx.doi .org/10.1016/j·jcp.2016.05.060

[62] S. Aoi, H. Fujiwara, 3d finite-difference method using discontinuous grids, Bull. Seism. Soc. Am. 89 (4) (1999) 918-930.

[63] Y. Wang, J. Xu, G. Schuster, Viscoelastic wave simulation in basins by a variable-grid finite-difference method, Bull. Seism. Soc. Am. 91 (6) (2001) 1741-1749.

[64] J. Kristek, P. Moczo, M. Galis, Stable discontinuous staggered grid in the finite-difference modelling of seismic motion, Geophys. J. Int. 183 (3) (2010) 1401-1407. doi:10.1111/j.1365-246x.2010.04775.x. URL http://dx.doi.org/10.1111/j.1365-246x.2010.04775.x

[65] K. Mattsson, M. H. Carpenter, Stable and accurate interpolation operators for high-order multiblock finite difference methods, SIAM J. Sci. Comput. 32 (4) (2010) 2298-2320. doi:10.1137/090750068.

URL http://dx.doi.org/10.1137/090750068 